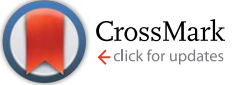

Cite this: RSC Adv., 2015, 5, 61440
Received 19th May 2015

Accepted 25th June 2015

DOI: $10.1039 / \mathrm{c} 5 \mathrm{ra09400c}$

www.rsc.org/advances

\section{Interpenetrated 3D porous scaffolds of silk fibroin with an amino and octadecyl functionalized hyaluronic acid}

\begin{abstract}
F. S. Palumbo, ${ }^{a}$ C. Fiorica, ${ }^{a}$ G. Pitarresi, ${ }^{\star a b c}$ S. Agnello ${ }^{a}$ and G. Giammona ${ }^{a b c}$
An ethylenediamine (EDA) and octadecylamine $\left(-\mathrm{C}_{18}\right)$ hyaluronic acid $(\mathrm{HA})$ derivative, named HA-EDA- $\mathrm{C}_{18}$, has been used for the production of interpenetrated composite biomaterials with silk fibroin. The peculiar ionic strength sensibility of this HA derivative allows the production of porous matrices without the need for chemical crosslinking. Scaffolds have been produced through a salt leaching procedure by exploiting the properties of silk fibroin and $\mathrm{HA}-\mathrm{EDA}-\mathrm{C}_{18}$ to physically crosslink when forced through a syringe loaded with $\mathrm{NaCl}$. The porosity of the sponges, comprised between $70-80 \%$, was dependent on the amount of each polymer and $\mathrm{NaCl}$ size distribution. Moreover, through FT-IR analysis, it has been evaluated how the blend composition influences the conformational changes of fibroin. This study demonstrates that $\mathrm{HA}-\mathrm{EDA}-\mathrm{C}_{18}$ induces the transition of silk fibroin to its $\beta$-sheet conformation. Moreover, the HA-EDA$\mathrm{C}_{18}$ weight fraction in composite sponges regulates the scaffold susceptibility to protease and hyaluronidase. FT-IR analysis and enzymatic hydrolysis studies suggest that the homogeneous interpenetration of polymers occurs. In vitro studies indicate that the presence of fibroin improves the viability and attachment of bovine chondrocytes.
\end{abstract}

\section{Introduction}

Interpenetrating biomaterials (IPNs) are "alloys" of crosslinked polymers where at least one is physically crosslinked to the other, and cannot be separated unless chemical bonds are broken. ${ }^{1-3}$ The interpenetration can be advantageous to impart to the resulting composite some peculiar characteristics that cannot be found in the materials formed by each single polymer or in the composite where interpenetration does not occurs. Silk fibroin, derived from the silkworm Bombyx mori, is a fibrous protein composed of two domains of 350 and $25 \mathrm{kDa}$ interconnected by thiol bridges. The protein presents a high content of the hydrophobic pentapeptide Gly-Ala-Gly-Ala-Ser that can assemble into $\beta$-sheet crystalline coils, thus allowing the formation of physically crosslinked hydrogels. Such a material has found a promising employ in regenerative medicine thanks to its advantageous mechanical and biological properties. ${ }^{4}$

Several examples of interpenetrating systems with natural or synthetic polymers and silk fibroin have been already described, because interpenetrating polymers can improve the fibroin transition from a random coil $\alpha$-helix to a crystalline $\beta$-sheet

${ }^{a}$ Dipartimento di Scienze e Tecnologie Biologiche Chimiche e Farmaceutiche, Sezione di Chimica e Tecnologie Farmaceutiche, Università degli Studi di Palermo, Via Archirafi 32, 90123, Palermo, Italy. E-mail: giovanna.pitarresi@unipa.it

${ }^{b}$ IBIM-CNR, Via Ugo La Malfa 153, 90146 Palermo, Italy

${ }^{c}$ Institute of Biophysics at Palermo, Italian National Research Council, Via Ugo La Malfa 153, 90146 Palermo, Italy conformation..$^{5-7}$ In particular several studies have been performed on composites between fibroin and glycosaminoglycans, like hyaluronic acid (HA), and the effects of this interpenetration on the physical and biological properties of the protein have been evaluated. ${ }^{7-10}$ Silk fibroin/HA blend scaffolds promote cell proliferation, infiltration and extracellular matrix deposition as compared to fibroin scaffolds. However the use of unmodified hyaluronic acid for the production of composite fibroin/HA scaffolds shows some drawbacks such as fast diffusion from the matrix because of its high water affinity, fast hydrolysis ${ }^{8}$ and very low versatility to exploit scaffold processing technologies.

In order to overcome these problems, chemically crosslinked sponges of fibroin and hyaluronic acid have been designed for in vitro or in vivo cultures of chondrocytes, and neuronal, myocardial and staminal cells. ${ }^{\mathbf{1 1 , 1 2}}$ The aim of this work was to evaluate the properties and stability of a series of interpenetrating biomaterials obtained with fibroin and a HA derivative bearing both hydrophobic and amino pendant chains, here reported as $\mathrm{HA}-\mathrm{EDA}-\mathrm{C}_{18}$, without the need for chemical crosslinking. Indeed, hydrophobic functionalization (obtained by introducing an octadecylamine portion, $\mathrm{C}_{18}$, as a pendant chain) allows the formation of a physically crosslinked scaffold unlike the starting $\mathrm{HA}^{13}{ }^{13}$ while the protonation of the pendant free amino groups (introduced as an ethylenediamine portion, EDA) improved water affinity. HA-EDA-C ${ }_{18}$ was physically interpenetrated with fibroin through a salt leaching process that allowed porous scaffolds to be obtained. Moreover, 
with the aim to investigate the effect of HA-EDA- $\mathrm{C}_{18}$ interpenetration on silk fibroin conformational changes, the scaffolds were not exposed to water vapor or an alcohol annealing treatment commonly used to cause conformation transition of silk fibroin from $\alpha$-helix to $\beta$-sheet. Different weight ratios of fibroin/ HA-EDA- $\mathrm{C}_{18}$ were employed and the resulting scaffolds were characterized in terms of porosity and resistance to enzymatic hydrolysis. The transition of fibroin from a silk I to a silk II conformation (i.e. from a random coil $\alpha$-helix to a crystalline $\beta$ sheet conformation) was detected through FT-IR analysis by studying the influence of the presence of salt and HA-EDA-C ${ }_{18}$. The obtained scaffolds were also characterized in terms of suitability for bovine chondrocyte entrapment and culturing.

\section{Experimental section}

\section{Materials and methods}

High molecular weight hyaluronic acid (2.01 kDa) was purchased from Altergon (Italy). Low molecular weight HA $\left(M_{\mathrm{w}} 220 \mathrm{kDa}\right.$, polydispersity index 1.8) and the tetrabutylammonium salt of hyaluronic acid (HA-TBA) were produced as reported elsewhere ${ }^{14,15}$ Octadecylamine $\left(\mathrm{C}_{18}\right)$, tetrabutylammonium hydroxide (TBA-OH), testicular hyaluronidase (HAase) (1040 $\mathrm{U} \mathrm{mg}^{-1}$ ), bis(4-nitrophenyl) carbonate (4-NPBC), picrylsulfonic acid solution (2,4,6-trinitrobenzenesulfonic acid-TNBS), $t$-butyl-carbazate, protease XIV, and hexamethyldisilazane were purchased from Sigma-Aldrich (Milano, Italy). Ethylenediamine (EDA) and anhydrous dimethylsulfoxide (DMSO) were all from Fluka (Milano, Italy). The regenerated silk fibroin from Bombyx mori was a generous gift from "Stazione Sperimentale per la Seta" (Milano, Italy). $\mathrm{NaCl}$ was ground with a mortar and sieved using a mechanic sieve.

${ }^{1} \mathrm{H}-\mathrm{NMR}$ spectra were obtained with a Bruker AC-300 instrument. SEC analysis was performed using a multidetector SEC system equipped with a Waters 600 pump, a Waters 410 Refractive Index Detector and a linear column from Waters (particle size $5 \mu \mathrm{m}$ ). The calibration curve was determined by using standards of HA purchased from Hyalose (USA). The elution medium was $200 \mathrm{mM}$ phosphate buffer ( $\mathrm{pH} 6.5) /$ $\mathrm{MeOH} 90: 10(\mathrm{v} / \mathrm{v})$, with a flow rate of $0.6 \mathrm{ml} \mathrm{min}^{-1}$ at $35{ }^{\circ} \mathrm{C}$. UV measurements were carried out by using a Shimadzu UV2401PC spectrophotometer. FT-IR analysis was performed using a Bruker Alpha instrument. Scanning electron microscopy (SEM) images were recorded by using a Philips XL30 ESEM scanning electron microscope; the samples were freeze-dried, freeze fractured, gold coated and finally observed.

\section{Synthesis of HA-EDA- $\mathrm{C}_{18}$}

Aliquots of HA-TBA ( $1 \mathrm{~g})$ were dissolved in DMSO $(88 \mathrm{ml})$ at $40{ }^{\circ} \mathrm{C}$ and activated by adding the proper amount of 4 -NPBC, dissolved in $8 \mathrm{ml}$ of DMSO, to satisfy the molar ratio of 4-NPBC/ HA-TBA repetitive units equal to 0.7 or 1 . After $4 \mathrm{~h}$, each solution was transferred into a bath at $60{ }^{\circ} \mathrm{C}$, then an appropriate amount of octadecylamine $\left(\mathrm{C}_{18}\right)$, previously dissolved in $4 \mathrm{ml}$ of DMSO at $70{ }^{\circ} \mathrm{C}$, was added. Four different molar ratios of octyadecylamine/HA-TBA repetitive units were employed, i.e.
$0.2,0.35,0.7$ and 1.0, for samples a, b, c and d, respectively. After $24 \mathrm{~h}$, each sample was cooled to $40{ }^{\circ} \mathrm{C}$, then an excess of ethylenediamine (EDA) was added according to a molar ratio of EDA/HA-TBA repetitive units equal to 7 or $10 .^{16}$ The reaction was performed further for $3 \mathrm{~h}$, then stopped by adding $1 \mathrm{ml}$ of a saturated $\mathrm{NaCl}$ aqueous solution. Each polymer was isolated by precipitation in an excess of diethyl ether/chloroform in a ratio of $1: 1 \mathrm{v} / \mathrm{v}$, and washed in the same solvent for 4-5 times, then with ethanol/water $(8: 2 \mathrm{v} / \mathrm{v})$ and finally with ethanol. The final products appearing as white powders were dried under vacuum, then characterized by ${ }^{1} \mathrm{H}-\mathrm{NMR}$ to determine the $\mathrm{C}_{18}$ molar functionalization and by colorimetric assay with $\mathrm{TNBS}^{17}$ to determine the EDA molar functionalization.

A typical ${ }^{1} \mathrm{H}-\mathrm{NMR}$ spectrum of HA-EDA- $\mathrm{C}_{18}$ in $\mathrm{D}_{2} \mathrm{O} / \mathrm{THFd}_{8}$ $2: 1$ showed: $\delta 0.99\left(-\mathrm{CH}_{3}\right.$ octadecylamine); $\delta 1.5\left(-\mathrm{CH}_{2}-\right.$ $\left(\mathrm{CH}_{2}\right)_{16}-\mathrm{CH}_{3}$ octadecylamine); $\delta 1.9\left(\mathrm{CH}_{3}\right.$ methyl group of the HA $N$-acetylglucosaminic portion) and $\delta 3.3-4.0$ (disaccharide pyranosil of $\mathrm{HA}$ ). In particular, the degree of $\mathrm{C}_{18}$ functionalization was calculated by comparing peaks at $\delta 0.99$ and 1.5, attributable to the $\mathrm{CH}_{3}$ and $\mathrm{CH}_{2}$ of the octadecylamine with the peak at 1.9 attributable to HA. The derivatization degrees in $\mathrm{C}_{18}$ and EDA were expressed as mean values \pm standard deviation.

\section{Preparation of the porous scaffolds}

Solutions of fibroin and HA-EDA-C $\mathbf{1 8}_{\mathbf{1 8}}$. Degummed silk fibroin was dispersed as reported elsewhere ${ }^{18}$ in a ternary solution composed of water, $\mathrm{CaCl}_{2}$ and ethanol (in a ratio of $41.5: 32: 26.5 \mathrm{v} / \mathrm{v}$ ) at $60{ }^{\circ} \mathrm{C}$ then dialyzed against water. The aqueous solution was concentrated at $6 \% \mathrm{w} / \mathrm{v}$ by performing a dialysis (using a tube with a cut-off of $3.5 \mathrm{kDa}$ ) against a dispersion of PEG (10 kDa). ${ }^{8}$

The HA-EDA- $\mathrm{C}_{18}$ derivative was dispersed in a water/THF solution (ratio $2: 1 \mathrm{v} / \mathrm{v}$ ) at $6 \% \mathrm{w} / \mathrm{v}$. Porous tridimensional scaffolds were produced using silk fibroin/HA-EDA- $\mathrm{C}_{18}$ mixtures and $\mathrm{NaCl}$ as a porogen. Known volumes of starting polymer solutions were mixed and homogenized through slow agitation and finally employed to fill $1 \mathrm{ml}$ syringes preloaded with $\mathrm{NaCl}$ having a specific size distribution. After $24 \mathrm{~h}$, the hydrogels formed inside the syringes were cut to produce cylindrical pieces with a volume of $0.5 \mathrm{ml}$.

Hydrogels were exhaustively washed in DPBS pH 7.4 to assure the complete diffusion of the porogen and finally freezedried. Fifteen different batches of scaffolds were produced by modulating the volumetric ratios of the silk fibroin and HAEDA- $\mathrm{C}_{18}$ solutions employed and the specific size distribution of $\mathrm{NaCl}$. In particular the volumetric ratios of fibroin/HA-EDA$\mathrm{C}_{18}$ employed were 100/0, 75/25, 50/50, 25/75, 0/100 (and the corresponding scaffolds were indicated as samples A, B, C, D and $\mathrm{E}$, respectively). For each scaffold three different $\mathrm{NaCl}$ size distributions were used, i.e. 180-212 $\mu \mathrm{m}, 212-250 \mu \mathrm{m}$ and 250$500 \mu \mathrm{m}$.

SEM analysis and porosity measurements. The obtained scaffolds were frozen in liquid nitrogen, then cross fractured. The inner section was gold sputtered and observed by SEM. Representative images were compared to have a qualitative porosity assessment. 
The porosity of sponges $(\varnothing 1 \mathrm{~cm}, h 1 \mathrm{~cm})$ was evaluated using the liquid displacement method ${ }^{19}$ where each sponge of weight $W$ was immersed in a graduated cylinder containing a known volume $\left(V_{1}\right)$ of hexane. The system was then placed under vacuum to remove the entrapped air and the total volume of solvent and scaffold was recorded as $V_{2}$. The scaffold was removed from the solvent and the residual hexane was measured as $V_{3}$.

Then the porosity was calculated using the following expression:

$$
\text { Porosity }=\left(V_{1}-V_{2}\right) /\left(V_{2}-V_{3}\right)
$$

Measurements were performed on 6 samples for each batch of scaffold and the results expressed as porosity $\%, \pm$ standard deviation.

Fourier transform infrared spectroscopy analysis. The fibroin secondary structure was evaluated via FT-IR spectroscopy. ${ }^{7}$ The spectra of the scaffolds produced as reported in the previous section were compared with the spectra of the fibroin/ HA-EDA-C ${ }_{18}$ scaffold obtained without the use of the porogen. Such scaffolds were produced by introducing the fluid fibroin/ HA-EDA- $\mathrm{C}_{18}(75 / 25,50 / 50,25 / 75)$ mixture into the syringe and waiting $24 \mathrm{~h}$ to allow hydrogel formation. The matrices were then washed with DPBS pH 7.4 and finally freeze-dried. The fibroin control was instead produced by precipitating the fibroin solution into an excess of acetone, and the starting HAEDA- $\mathrm{C}_{18}$ was used as the control. The samples prepared without porogen were indicated as $\mathrm{A}^{*}, \mathrm{~B}^{*}, \mathrm{C}^{*}, \mathrm{D}^{*}$ and $\mathrm{E}^{*}$.

Swelling and enzymatic hydrolysis studies. The swelling of scaffolds (obtained with $\mathrm{NaCl} 212-250 \mu \mathrm{m}$ ) was evaluated after immersion at $37^{\circ} \mathrm{C}$ in $4 \mathrm{ml}$ of DPBS pH 7.4 for 2,24 or $48 \mathrm{~h}$. The air entrapped into each scaffold was removed under vacuum. After each time, the scaffolds were withdrawn using tweezers and gently treated with blotting paper to remove the unabsorbed excess DPBS and weighed to calculate the ratio of swelling, $q$ (weight swollen/weight dried).

The stability of the scaffolds to the enzymatic activity was assayed by evaluating their weight loss $\%$ and the release of uronic acid. In particular, the scaffolds obtained with $\mathrm{NaCl} 212-$ $250 \mu \mathrm{m}$ were incubated at $37^{\circ} \mathrm{C}$ in $4 \mathrm{ml}$ of DPBS pH 7.4 in the presence of hyaluronidase (HAase) $50 \mathrm{U} \mathrm{ml}^{-1}$ (excluding batch A) or protease XIV $10 \mathrm{U} \mathrm{ml}^{-1}$ (excluding batch E).

The weight loss $\%$ was evaluated by comparing the weight of the freeze-dried scaffold obtained after washing with the starting scaffold weight. The amount of uronic acid released after the enzymatic treatment was evaluated using a carbazole assay. ${ }^{20}$ Each experiment was repeated three times and the results were expressed as mean \pm standard deviation.

In vitro chondrocyte culture in the scaffolds. Bovine chondrocytes were isolated from bovine cartilage following a procedure reported elsewhere. ${ }^{13}$ The primary chondrocytes were stored in liquid nitrogen and then expanded until passage four before their use. Cylindrical scaffolds $(\varnothing 5 \mathrm{~mm}, h 5 \mathrm{~mm})$ obtained by using $\mathrm{NaCl} 212-250 \mu \mathrm{m}$ as a porogen were loaded with $100 \mu \mathrm{l}$ of a cellular suspension in complete DMEM $(4 \times$
$10^{5}$ ) into 96 well plates. After $1 \mathrm{~h}$ of incubation, $1 \mathrm{ml}$ of DMEM was added for each well and exchanged using a fresh medium every two days. The cell viability was evaluated after 1, 7 and 14 days of incubation using the mitochondrial activity MTS assay [3-(4,5-dimethylthiazol-2-yl)-5-(3-carboxymethoxyphenyl)-2-(4sulfophenyl)-2H-tetrazolium] (CellTiter 96® AQueous One Solution Cell Proliferation, PROMEGA). In particular, at the scheduled times, the samples were incubated for $4 \mathrm{~h}$ at $37{ }^{\circ} \mathrm{C}$ and $5 \% \mathrm{CO}_{2}$ with a $20 \% \mathrm{v} / \mathrm{v}$ solution of MTS in supplemented DMEM. After this time, the absorbance at $492 \mathrm{~nm}$ of the solution was evaluated with a multiplate spectrophotometer reader (Multiskan EX Thermo Scientific). The viability of the cells cultured in the well without the sample was used as the positive control.

Evaluation of the chondrocyte shape on scaffolds via SEM analysis. The chondrocytes on scaffolds were observed by SEM analysis after 1, 7 and 14 days from their loading. In particular, to perform the experiment, the scaffolds were first fixed with formalin $4 \% \mathrm{v} / \mathrm{v}$, dehydrated through sequential immersion in ethanolic solutions $(30,50,70,90 \% \mathrm{v} / \mathrm{v}$, pure ethanol), then treated with hexamethyldisilazane overnight, and finally dried under a hood. ${ }^{21}$ The samples were then gold sputtered and observed.

Statistical analysis. All results are reported as mean \pm standard deviation and, when applicable, a statistical analysis for significance was performed by the means of Student's $t$-test, using Microsoft Excel's statistical function for $t$-tests, assuming an unequal variance and two tailed distribution; values of $p<$ 0.05 were considered statistically significant.

\section{Results and discussion}

Three subsequent chemical steps, without intermediate isolation and purification passage were performed to obtain the final HA derivative as reported in Fig. 1. This HA derivative was synthesized as reported elsewhere through the activation of $\mathrm{N}$ acetylglucosamine hydroxyl groups of HA with bis(4nitrophenyl)carbonate (4-NPBC) and the sequential addition of octadecylamine and ethylenediamine..$^{13,16}$

It has been found that the functionalization degrees in $\mathrm{C}_{18}$ and EDA are dependent on the molar ratios between the amine and HA repetitive units. By increasing the molar ratios of octadecylamine/HA-TBA repetitive units, it was possible to obtain a range of functionalization between 8 and $26 \mathrm{~mol} \%$ (Table 1). In addition, the functionalization in EDA decreases with increasing $\mathrm{C}_{18}$ functionalization. As observed for similar hydrazide HA derivatives, ${ }^{22}$ it seems that the increase in $\mathrm{C}_{18}$ chains reduces the efficiency of functionalization of the unreacted $p$-nitrophenol activated $N$-acetylglucosamine units.

The obtained derivatives show a different affinity towards aqueous media. Derivative a is soluble in water and in aqueous solutions with ionic strength in the range 0.1-0.3 M. Oppositely, derivatives $\mathrm{b}$ and $\mathrm{c}$ are soluble in water and sensitive even to a slight increase in the ionic strength which triggers their desolvation and precipitation. Derivative $d$ has a slight affinity to water where it produces micro-hydrogels. This behavior is consistent with the increase in hydrophobic functionalization 


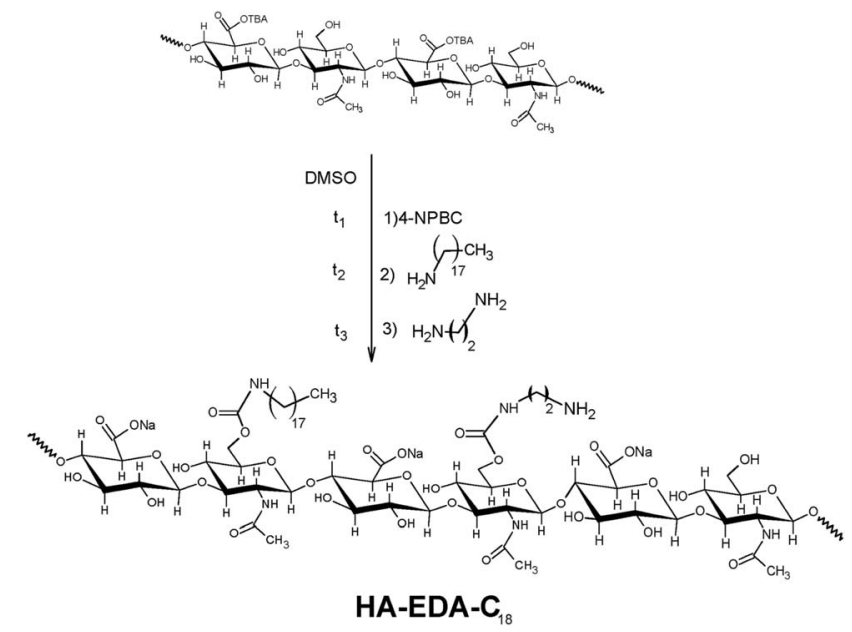

Fig. 1 Scheme of the functionalization procedure to obtain HA-EDA$C_{18} ; t_{1}, t_{2}, t_{3}$ are the times of reaction reported in Table 1.

with $\mathrm{C}_{18}$ chains and the decrease in EDA functionalization observed from sample a to sample d.

The peculiar behavior of derivatives $b$ and $c$ can be exploited for the production of physically assembled matrices. Reasonably, when a concentrated solution of the derivative $b$ or $c$ is forced through a syringe filled with solid $\mathrm{NaCl}$, upon contact, desolvation occurs, leading to the formation of a hydrophobically assembled hydrogel. This distinctive behavior was exploited here to produce interpenetrating composites between derivative $\mathrm{b}$ and silk fibroin, by using $\mathrm{NaCl}$ as a porogen. Two solutions of HA-EDA- $\mathrm{C}_{18}$ and silk fibroin were prepared, each with $6 \% \mathrm{w} / \mathrm{v}$. A water/THF mixture was used to dissolve HAEDA- $\mathrm{C}_{18}$; the use of THF was necessary to produce a HA-EDA$\mathrm{C}_{18}$ solution with the appropriate fluidity to be mixed and injected through the salt loaded syringe. The aqueous silk fibroin solution $(6 \% \mathrm{w} / \mathrm{v})$ was prepared as reported in the Experimental section. ${ }^{23,24}$ The starting solutions and mixtures between them were forced through a syringe loaded with $\mathrm{NaCl}$ as represented in Fig. 2 .

Three different dimensional ranges of porogen (212-180 $\mu \mathrm{m} ; 250-212 \mu \mathrm{m} ; 500-250 \mu \mathrm{m})$ were used to evaluate the influence on the final scaffold porosity. For each batch of employed porogen, five fibroin/HA-EDA-C 18 compositions were produced by mixing proper ratios of the starting solutions. Table 2 reports the scaffold compositions produced in our experiments.

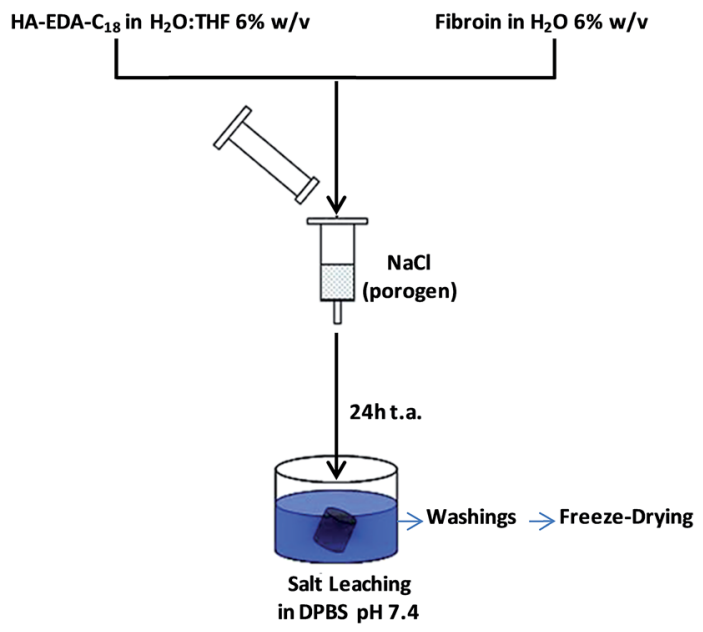

Fig. 2 Scheme of the procedure employed for scaffold production.

SEM analysis was performed to gain qualitative information about the microscopic appearance of the produced scaffolds.

Pictures A1-A3 in Fig. 3 show the inner surface of the scaffolds of silk fibroin alone obtained using $\mathrm{NaCl}$ with different dimensional distributions. It is already known that the contact of a silk fibroin solution with $\mathrm{NaCl}$ causes the transition of the protein from an $\alpha$-helix to a crystalline $\beta$-sheet with consequent gel formation. The use of salt with higher dimension seems to produce a matrix with better pore interconnection (mean pore of $100 \mu \mathrm{m}$ ) according to data reported elsewhere. ${ }^{25}$ Scaffolds of $\mathrm{HA}-\mathrm{EDA}-\mathrm{C}_{18}$ alone with a more homogeneous pore interconnection have been produced using the intermediate porogen dimension (see picture E2). Instead, for all batches containing both fibroin and HA-EDA- $\mathrm{C}_{18}$, no significant difference in pore interconnection has been found by varying the porogen size.

The quantitative porosity evaluation performed with the volume displacement method showed that all sponges have a

Table 2 Ratios between the fibroin and HA-EDA- $\mathrm{C}_{18}$ solutions employed for the production of scaffolds

\begin{tabular}{lrrrrr}
\hline & A & B & C & D & \multicolumn{1}{c}{ E } \\
\hline Silk fibroin & 100 & 75 & 50 & 25 & 0 \\
HA-EDA-C $_{18}$ & 0 & 25 & 50 & 75 & 100
\end{tabular}

Table 1 Batches of the produced $\mathrm{HA}-\mathrm{EDA}-\mathrm{C}_{18}$. The molar ratios of the reagents and HA-TBA repetitive units, with the reaction times and molar derivatization degrees (DD\%) in $\mathrm{C}_{18}$ and EDA are reported. DD\% are expressed as mean value of three different batches produced for each employed reaction condition

\begin{tabular}{|c|c|c|c|c|c|c|}
\hline HA-EDA-C 18 & $\begin{array}{l}\text { Mol 4-NPBC/mol } \\
\text { UR HA-TBA }\end{array}$ & $\begin{array}{l}\mathrm{Mol} \mathrm{C}_{18}-\mathrm{NH}_{2} / \mathrm{mol} \\
\text { UR HA-TBA }\end{array}$ & Mol EDA/mol HA-TBA & Time 1, 2, 3 (h) & $\mathrm{DD} \mathrm{C}_{18} \%$ & DD EDA\% \\
\hline $\mathrm{a}$ & 0.7 & 0.2 & 7 & $4,24,3$ & $8 \pm 0.6$ & $36 \pm 1.0$ \\
\hline c & 0.7 & 0.7 & 7 & $4,24,3$ & $17 \pm 2.0$ & $16 \pm 0.3$ \\
\hline d & 1 & 1 & 10 & $4,24,3$ & $26 \pm 3.4$ & $10 \pm 2.9$ \\
\hline
\end{tabular}




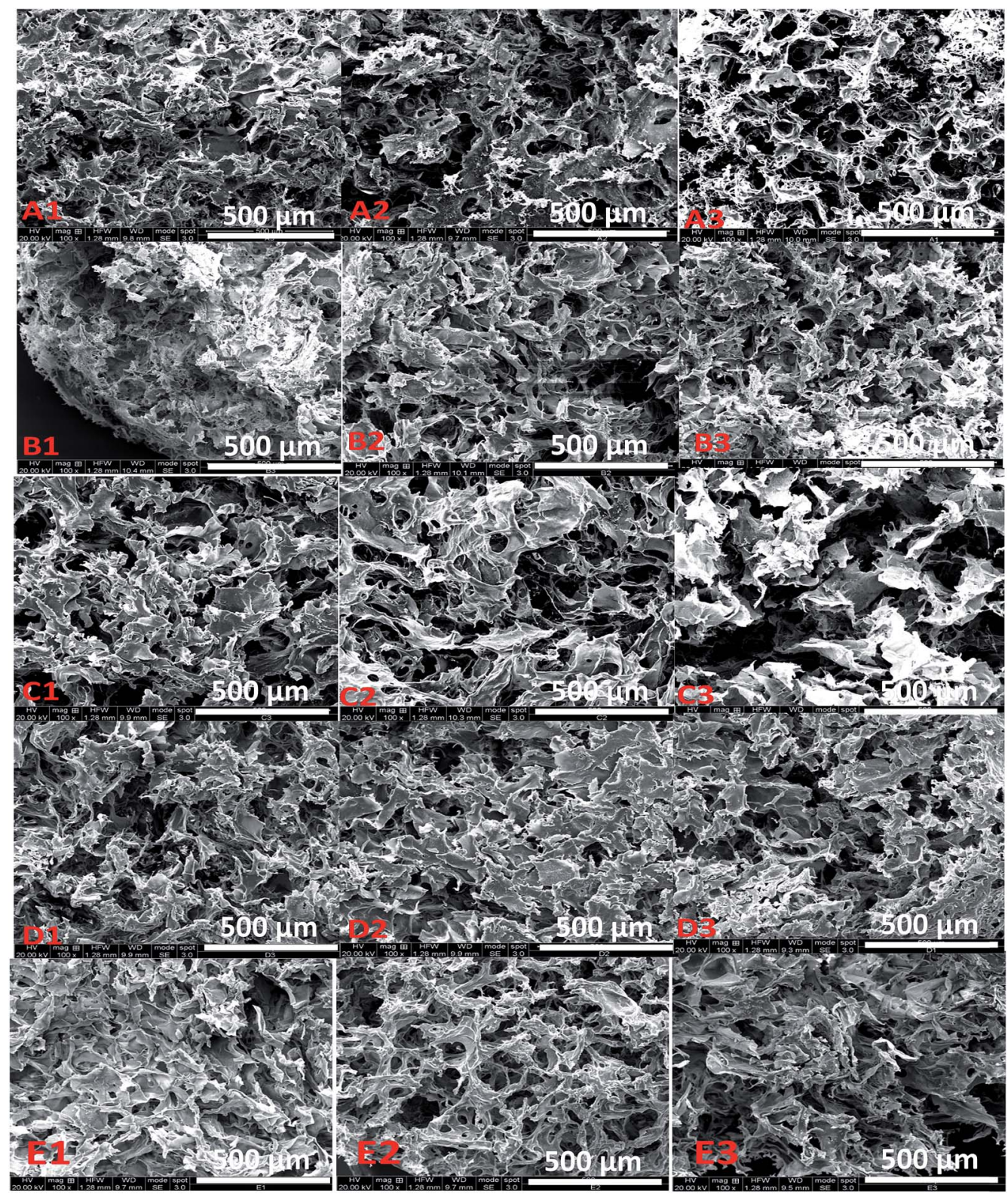

Fig. 3 SEM pictures of the porous sponges from the $A$ ( $100 \%$ of fibroin) to $E\left(100 \%\right.$ of HA-EDA- $C_{18}$ ) samples by using a porogen with different dimensional distributions (1: $\mathrm{NaCl} 180-212 \mu \mathrm{m} ; 2$ : $\mathrm{NaCl} 212-250 \mu \mathrm{m} ; 3: \mathrm{NaCl} 250-500 \mu \mathrm{m}$ ).

porosity higher than $70 \%$ (see Table 3 ). Samples A (fibroin alone matrices) showed a porosity that increases with increasing salt dimensional range as reported elsewhere ${ }^{25}$ indeed, batch 3 of $\mathrm{NaCl}(250-500 \mu \mathrm{m})$ produces a porosity higher than $80 \% .{ }^{26}$ No statistically significant differences in porosity were observed for the silk fibroin/HA-EDA- $\mathrm{C}_{18}$ (samples B-D) composites for all batches of $\mathrm{NaCl}$.

The study of the secondary structure of fibroin was performed via FT-IR analysis to evaluate the possible influence of HA-EDA- $\mathrm{C}_{18}$ on the transition of fibroin from an $\alpha$-helix to a $\beta$ sheet conformation. In particular, the shifts to lower wavenumbers of the peaks related to the amide I and II of fibroin (in the ranges $1650-1655$ and $1540-1550 \mathrm{~cm}^{-1}$, respectively) and the appearance of the shoulder at $1260 \mathrm{~cm}^{-1}$ are proof of a fibroin conformation change to a $\beta$-sheet structure. ${ }^{7,10}$ In order to evaluate the effect of HA-EDA- $\mathrm{C}_{18}$ on the fibroin conformational changes only, annealing treatment was not performed. ${ }^{27}$ As already demonstrated elsewhere, ${ }^{25}$ contact with $\mathrm{NaCl}$ causes the conformational change of fibroin, in Fig. $4 \mathrm{~A}$ it is indeed

Table 3 Porosity \% of the samples obtained using different $\mathrm{NaCl}$ dimensional distributions. Batch 1: $180-212 \mu \mathrm{m}$; batch 2: 212-250 $\mu \mathrm{m}$; batch 3: $250-500 \mu \mathrm{m}$

\begin{tabular}{llllll}
\hline NaCl batch & A & B & C & D & E \\
\hline 1 & $75 \pm 3$ & $74 \pm 2$ & $75 \pm 3$ & $75 \pm 2$ & $78 \pm 1$ \\
2 & $78 \pm 2$ & $76 \pm 3$ & $78 \pm 4$ & $76 \pm 2$ & $80 \pm 2$ \\
3 & $84 \pm 1$ & $75 \pm 4$ & $73 \pm 2$ & $73 \pm 1$ & $76 \pm 1$
\end{tabular}


possible to observe a shift to lower wavenumbers of amides I and II and the appearance of a peak at $1260 \mathrm{~cm}^{-1}$ for sample A, as compared to sample A* (prepared without porogen). The direct comparison of the FT-IR spectra of sample $B$ and sample $\mathrm{B}^{*}$ (obtained without salt leaching) and with the control made of a powder obtained by mixing silk fibroin and HA-EDA- $\mathrm{C}_{18}$ allows evaluation of how interactions between both polymers and porogen influence the fibroin conformational transition. The shift of peaks attributable to amide I and II and the appearance of the sharp shoulder at $1261 \mathrm{~cm}^{-1}$ (see Fig. 4B) clearly indicate that in both samples, i.e. scaffolds $\mathrm{B}$ and $\mathrm{B}^{*}$, the transition of fibroin to a $\beta$ sheet conformation occurs. Several studies have reported a possible positive effect of HA on fibroin in accelerating the protein conformational transition, even if results have often been controversial. However, all experiments confirm that HA does not promote the folding of silk fibroin but at best it can speed up the $\beta$-sheet formation when an annealing treatment is employed. ${ }^{7,10}$ Besides, other studies revealed how amphiphilic molecules such as surfactants can trigger a faster $\beta$ sheet formation in silk fibroin aqueous solutions, ${ }^{8,9}$ thus suggesting how they can be used as gelling additives. This effect has been attributed to the interaction of the surfactant with fibroin, through hydrogen bonding and hydrophobic interactions that accelerate protein $\alpha$-helix unfolding and hydrophobic coil aggregation into a $\beta$-sheet conformation. Therefore, considering the amphiphilic character of HA-

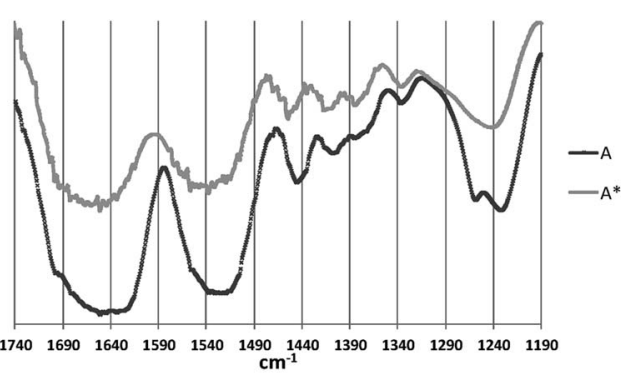

(A)

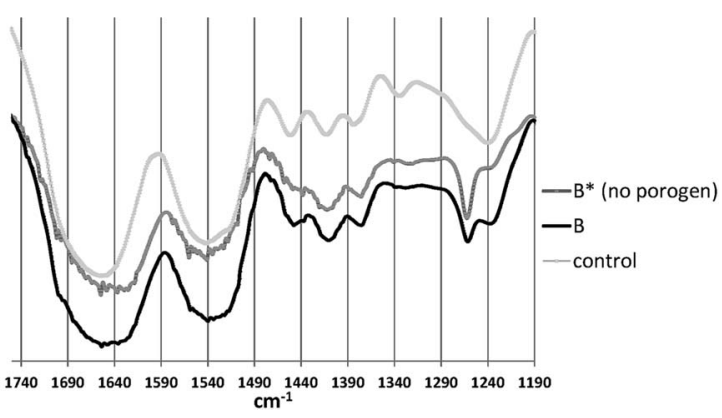

(B)

Fig. 4 FT-IR spectra in the wavelength range comprising the peaks of amides I, II and III of fibroin (sample A) and the silk fibroin/HA-EDA$\mathrm{C}_{18}$ composites $\mathrm{B}$. Panel A: overlapping of the FT-IR spectra of sample $A$ and silk fibroin control $A *$. Panel $B$ : overlapping of the FT-IR spectra of samples $B, B *$ (scaffold $B$ obtained by spontaneous gelation of silk fibroin/HA-EDA- $C_{18} 75 / 25$ without the use of porogen) and a control made of a physical mixture of sample $A^{*}$ and the HA-EDA-C ${ }_{18}$ powder.
EDA- $\mathrm{C}_{18}$, it is reasonable to suppose that this HA derivative causes a surfactant-like effect on the fibroin conformation transition.

Interestingly, the intensity of the peak at $1261 \mathrm{~cm}^{-1}$ is higher for composite $\mathrm{B}^{*}$ obtained without the use of porogen. Indeed, while in the presence of porogen the HA-EDA- $\mathrm{C}_{18}$ derivative undergoes self-assembly just after syringe loading, because of hydrophobic chain desolvation. When the hydrogel is formed without the use of a porogen, a more complete and prolonged interaction between fibroin and $\mathrm{HA}-\mathrm{EDA}-\mathrm{C}_{18}$ is allowed, thus producing a more efficient $\beta$-sheet transition.

The influence of blend composition on swelling behavior was evaluated in DPBS $\mathrm{pH} 7.4$ as a function of time. As reported in Fig. 5A, an increased amount in HA-EDA- $\mathrm{C}_{18}$ increases the scaffold's water affinity and consequently the swelling ratio, in accordance with the hydrophilic character of hyaluronic acid. In particular, composite samples B-D swelled significantly more than sample A (100\% fibroin), but less than sample E $(100 \%$ HA-EDA-C $\left.{ }_{18}\right)(p<0.05)$. Instead, no statistically significant differences were found between the swelling values of scaffolds $\mathrm{B}$ and $\mathrm{C}(p=0.06), \mathrm{C}$ and $\mathrm{D}(p=0.2)$.

The scaffold resistance towards enzymatic hydrolysis in the presence of protease XIV or hyaluronidase (HAase) has also been evaluated. In the presence of protease XIV (see Fig. 5B and $5 \mathrm{D}$ ) a very fast hydrolysis of the fibroin sponge (sample A) occurs already after $2 \mathrm{~h}$ of incubation as confirmed by the data of weight loss \%. This hydrolysis is already significantly reduced for sponge $\mathrm{B}$ (where $25 \%$ of $\mathrm{HA}-\mathrm{EDA}-\mathrm{C}_{18}$ has been blended) with a weight loss \% after $2-48 \mathrm{~h}$ ranging from 45 to $79 \%$. The fast fibroin hydrolysis is in accordance with the missed annealing procedure; on the other hand, the significant decrease in fibroin loss for sample B if compared to sample A ( $p=0.0003$ after $24 \mathrm{~h}$ ) can be related to the positive effect of the $\mathrm{HA}-\mathrm{EDA}-\mathrm{C}_{18}$ derivative on the fibroin crystallinity as detected by FT-IR analysis.

After $48 \mathrm{~h}$, even sample D having a lower fraction of fibroin (25\%) undergoes a great loss of mass (about $60 \%$ ), thus indicating significant disaggregation of the matrix. This behavior caused by the protease activity even in sample D (having just $25 \%$ of fibroin) indicates a homogeneous interpenetration of polymers. Instead, in the presence of protease XIV, the amount of uronic acid (related to the release of HA soluble fragments) is low (10-20\% for all samples A-D) since this enzyme does not hydrolyze the hyaluronic acid backbone.

The data of the hydrolysis performed in the presence of HAase at $50 \mathrm{U} \mathrm{ml}^{-1}$ (see Fig. 5C and 5E) showed that only scaffold B undergoes a statistically significant loss of uronic acid after $48 \mathrm{~h}$ (about $30 \%$ of uronic acid lost, $p<0.05$, if compared to the other sponges that lose from 10 to $20 \%$ of uronic acid). The low amount of HA-EDA- $\mathrm{C}_{18}$ derivative in sample $\mathrm{B}$ probably reduces hydrophobic aggregation and increases the diffusion of HAase into the matrix and, as a consequence, its action on hyaluronic acid chains with the formation of soluble uronic acid. Besides, even if not associated with a significant increase in uronic acid release, a statistically significant increase in matrix disaggregation after $48 \mathrm{~h}$ of incubation was observed with the order: sample 


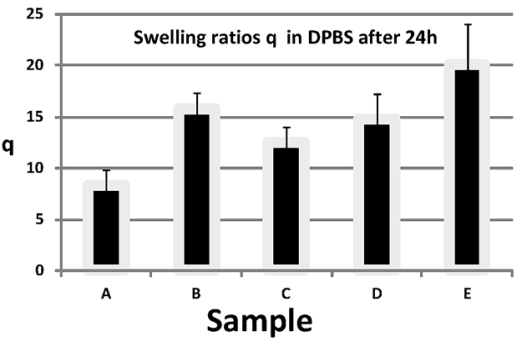

A
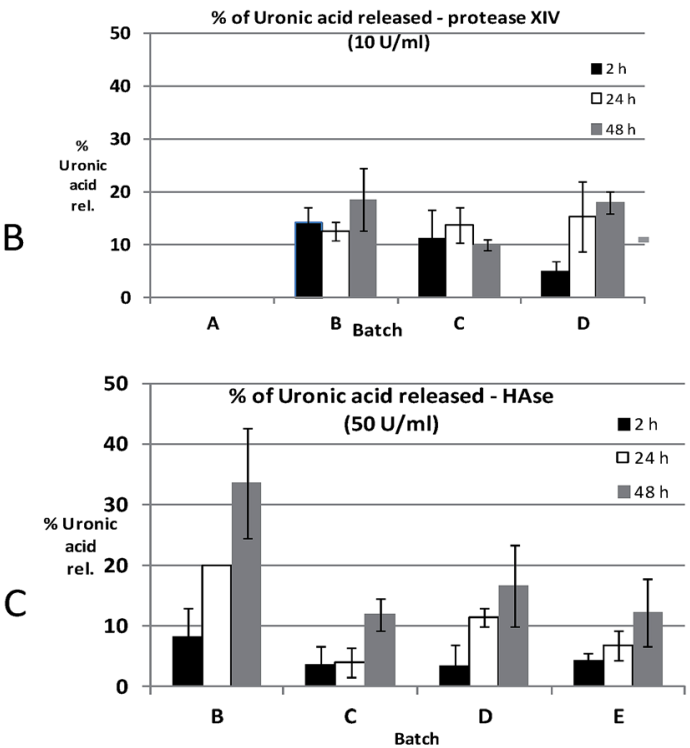

\begin{tabular}{|c|c|c|c|c|}
\hline & A & B & C & D \\
\hline $2 \mathrm{~h}$ & $100 \%$ & $45.93 \%$ & $26.22 \%$ & $29.33 \%$ \\
& & \pm 5.19 & \pm 5.97 & \pm 5.97 \\
\hline $24 \mathrm{~h}$ & $100 \%$ & $53.26 \%$ & $52.37 \%$ & $53.64 \%$ \\
& & \pm 6.03 & \pm 6.33 & \pm 4.85 \\
\hline $48 \mathrm{~h}$ & $100 \%$ & $79.06 \%$ & $44.59 \%$ & $57.08 \%$ \\
& & \pm 6.47 & \pm 7.09 & \pm 2.81 \\
\hline
\end{tabular}

\begin{tabular}{|c|c|c|c|c|}
\hline & $\mathrm{B}$ & $\mathrm{C}$ & $\mathrm{D}$ & $\mathrm{E}$ \\
\hline $2 \mathrm{~h}$ & $14.03 \%$ & $16.87 \%$ & $24.12 \%$ & $28.00 \%$ \\
& \pm 7.46 & \pm 8.05 & \pm 8.07 & \pm 1.19 \\
\hline $24 \mathrm{~h}$ & $9.97 \%$ & $8.84 \%$ & $52.22 \%$ & $28.35 \%$ \\
& \pm 3.98 & \pm 2.03 & \pm 6.04 & \pm 1.45 \\
\hline $48 \mathrm{~h}$ & $9.96 \%$ & $15.99 \%$ & $45.21 \%$ & $24.81 \%$ \\
& \pm 2.81 & \pm 3.09 & \pm 2.23 & \pm 0.46 \\
\hline
\end{tabular}

Fig. 5 (A) Swelling ratios expressed as $q$ (weight swollen/weight dry) of all samples A-E, after $24 \mathrm{~h}$, in DPBS pH 7.4. (B) \% of uronic acid released from samples $A-D$ in the presence of protease XIV $\left(10 \cup \mathrm{ml}^{-1}\right)$ after 2 , 24 and $48 \mathrm{~h}$; (C) \% of uronic acid released from samples $B-E$ in the presence of hyaluronidase $\left(50 \mathrm{U} \mathrm{ml}^{-1}\right)$ after 2, 24 and $48 \mathrm{~h}$; (D) weight loss \% from samples A-D in the presence of protease XIV $\left(10 \cup \mathrm{ml}^{-1}\right)$ after 2, 24 and $48 \mathrm{~h}$; (E) weight loss \% from samples B-E in the presence of hyaluronidase $\left(50 \mathrm{U} \mathrm{ml}^{-1}\right)$ after 2, 24 and $48 \mathrm{~h}$.

D > sample C > sample B (Fig. 5E). However, the loss \% of the scaffold in the presence of HAase is lower than that obtained in the presence of protease XIV; probably the hydrolysis of fibroin by protease produces soluble protein fragments that are lost by diffusion while HA-EDA- $\mathrm{C}_{18}$ hydrolysis, caused by HAase, produces a low amount of uronic acid.

The viability of bovine chondrocytes was evaluated by MTS assay comparing all produced scaffolds after cell incubation from 1 to 14 days (see Fig. 6). At day 1, fibroin scaffolds (sponge A) were able to retain the greatest percent of incubated cells, while sponges from B to E retained significantly less amounts of cells, that were found viable at the bottom of the wells according to a greater capacity of fibroin to improve the attachment and viability of cells. ${ }^{28}$ Scaffold E, composed of HA-EDA- $\mathrm{C}_{18}$ alone, maintained a stable viability of cells until 7 days of incubation but it falls to a lower value at day 14 $(p<0.01)$. This decrease in cell viability in the presence of sample $\mathrm{E}$ was however attributable to a progressive scaffold swelling that caused the loss of cells to the bottom of the vial; however these cells were found viable as assessed by the MTS assay and this result indicated low adherence of chondrocytes to the matrix of only HA-EDA-C ${ }_{18}$. For all fibroin containing scaffolds (samples A-D), a significant increase in cell viability occurs from day 1 to day 14 according to its known proliferative and adhesive properties even with chondrocytes ${ }^{29}$.

Chondrocyte distribution and attachment in each scaffold was observed via electron microscopy (SEM). Some selected pictures of scaffolds A-E at day 1 and of composites B-D after 7 and 14 days are reported in Fig. 7.

The cells already appear uniformly distributed inside each scaffold after 1 day of incubation (see arrows in images A-E of the upper panel in Fig. 7). However, for scaffold A (fibroin alone) cells appeared well attached and elongated (see image 1 of the upper panel in Fig. 7) whereas for scaffolds B, C, D and E, the chondrocytes are round shaped (see images $2-4$ of the upper panel in Fig. 7), thus suggesting a reduced adhesion with respect to scaffold A, according to the viability assay. After 7 and 14 days of incubation, also in composite scaffolds B, C and D, the cells appear well attached (see images in the lower panel of Fig. 7), thus suggesting that the presence of fibroin in these scaffolds allows the drawback of hyaluronic acid which, in spite of its chondrogenic effect, and because of its hydrophilic and polyanionic surface, does not favor cell attachment to be overcome. $^{30}$

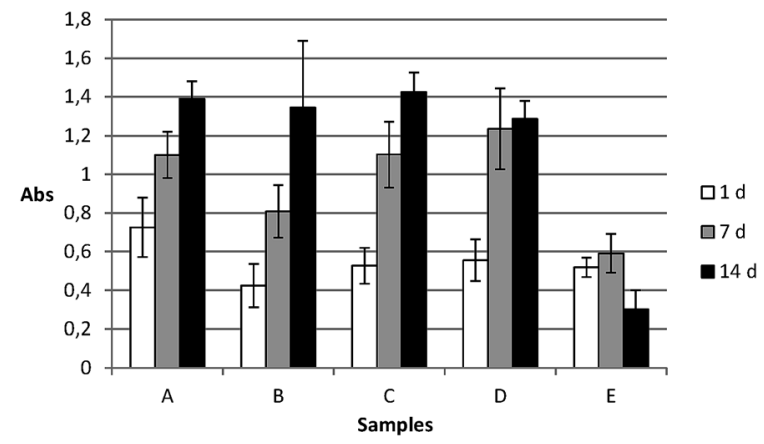

Fig. 6 Viability of chondrocytes on samples A-E evaluated by the MTS assay. The values are reported as Abs at $492 \mathrm{~nm}$ after 1, 7 and 14 days of culture. 

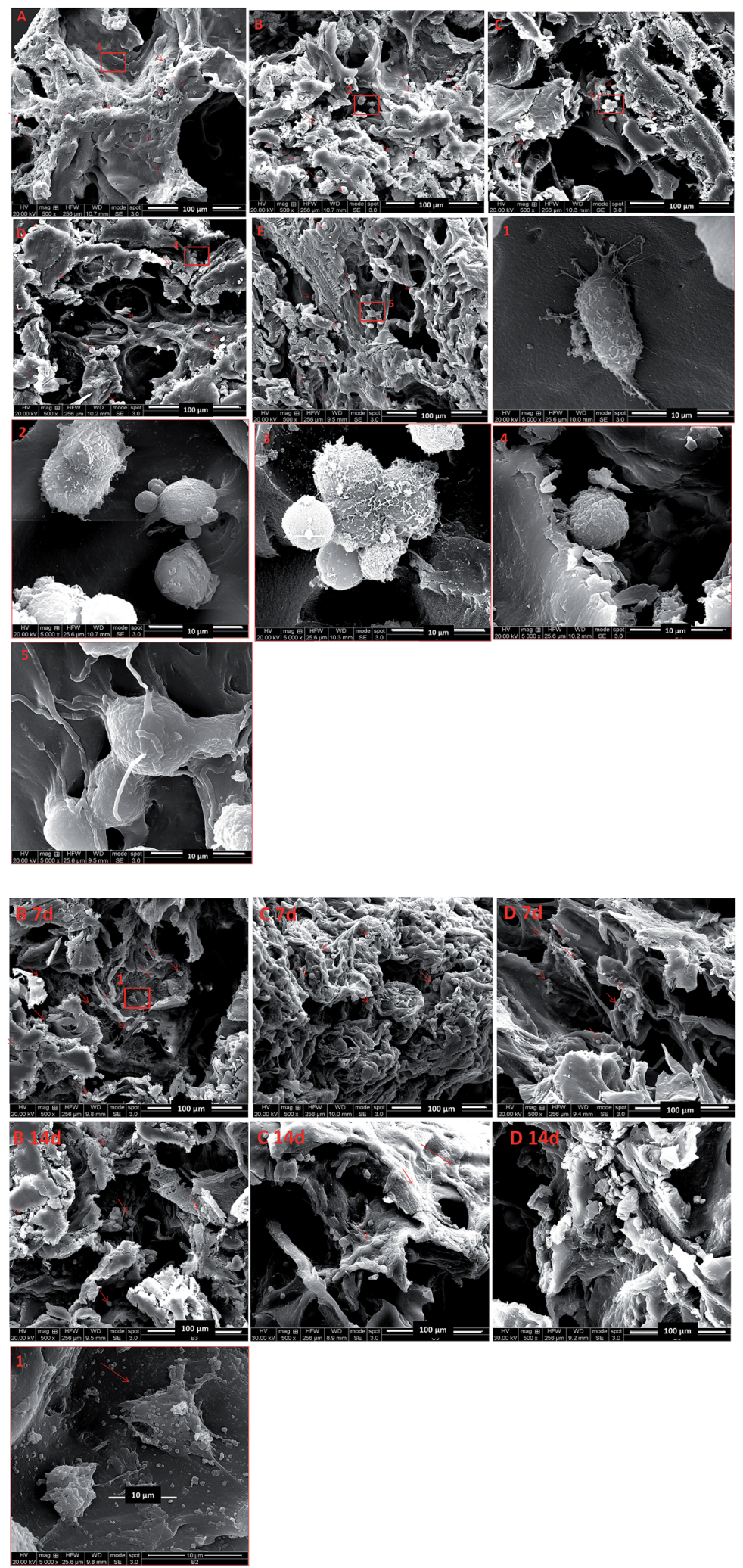

Fig. 7 Upper panel: SEM images of inner sections of scaffolds A-E after 1 day of incubation with chondrocytes (magnification $500 \times$ ) and related images 1-5 (magnification 5000x). Lower panel: SEM images of inner sections of scaffolds B-D after 7 and 14 days of incubation with chondrocytes (magnification 500x) and related image 1 (magnification 5000×). 


\section{Conclusions}

A hyaluronic acid (HA) functionalized derivative was synthesized bearing both ethylenediamine (EDA) and octadecyl $\left(-\mathrm{C}_{18}\right)$ chains as pendant portions. The affinity of HA-EDA- $\mathrm{C}_{18}$ derivatives towards aqueous medium is dependent on the derivatization degree in EDA and $\left(-\mathrm{C}_{18}\right)$ chains, being unsoluble in aqueous solutions with ionic strength in the range $0.1-0.3 \mathrm{M}$ by increasing the amount of hydrophobic chains.

Thanks to these peculiar properties, HA-EDA- $\mathrm{C}_{18}$ can be processed to obtain physically assembled porous matrices via a salt leaching technique. Interpenetrating porous scaffolds between HA-EDA- $\mathrm{C}_{18}$ and silk fibroin have been prepared in order to obtain composite scaffolds with appropriate properties. Enzymatic hydrolysis studies suggest a homogeneous entanglement between $\mathrm{HA}-\mathrm{EDA}-\mathrm{C}_{18}$ and silk fibroin in composite matrices.

The obtained data demonstrate that HA-EDA- $\mathrm{C}_{18}$ is stably integrated into the composite scaffolds with silk fibroin without the need to perform chemical crosslinking. The presence of HAEDA- $\mathrm{C}_{18}$ induced the fibroin transition from an $\alpha$-helix to a $\beta$ sheet conformation as confirmed by FT-IR analysis, while the presence of fibroin improves bovine chondrocyte attachment and proliferation. Therefore, the obtained results demonstrate the advantages of the combination between HA-EDA- $\mathrm{C}_{18}$ and silk fibroin, in preparing scaffolds for chondrocytes.

\section{References}

1 P. Matricardi, C. Di Meo, T. Coviello, W. E. Hennink and F. Alhaique, Adv. Drug Delivery Rev., 2013, 65, 1172-1187.

2 C. Vepari and D. L. Kaplan, Prog. Polym. Sci., 2007, 32, 9911007.

3 E. S. Dragan, Chem. Eng. J., 2014, 243, 572-590.

4 D. Kaplan, W. Wade Adams, B. Farmer and C. Viney, Silk Polymers, American Chemical Society, Washington, DC, 1993, vol. 544.

5 I.-S. Yeo, J.-E. Oh, L. Jeong, T. S. Lee, S. J. Lee, W. H. Park and B.-M. Min, Biomacromolecules, 2008, 9, 1106-1116.

6 N. Bhardwaj and S. C. Kundu, Biomaterials, 2012, 33, 28482857.

7 Z. Fan, F. Zhang, T. Liu and B. Q. Zuo, Int. J. Biol. Macromol., 2014, 65, 516-523.

8 C. Foss, E. Merzari, C. Migliaresi and A. Motta, Biomacromolecules, 2013, 14, 38-47.

9 X. Wu, J. Hou, M. Li, J. Wang, D. L. Kaplan and S. Lu, Acta Biomater., 2012, 8, 2185-2192.
10 M. Garcia-Fuentes, E. Giger, L. Meinel and H. P. Merkle, Biomaterials, 2008, 29, 633-642.

11 S. B. Rønning, M. E. Pedersen, P. V. Andersen and K. Hollung, Differentiation, 2013, 86, 13-22.

12 S. Yan, Q. Zhang, J. Wang, Y. Liu, S. Lu, M. Li and D. L. Kaplan, Acta Biomater., 2013, 9, 6771-6782.

13 F. S. Palumbo, C. Fiorica, M. Di Stefano, G. Pitarresi, A. Gulino, S. Agnello and G. Giammona, Carbohydr. Polym., 2014, 122, 408-416.

14 G. Pitarresi, F. S. Palumbo, R. Calabrese, E. F. Craparo and G. Giammona, J. Biomed. Mater. Res., Part A, 2008, 84, 413424.

15 C. Fiorica, G. Pitarresi, F. S. Palumbo, M. Di Stefano, F. Calascibetta and G. Giammona, Int. J. Pharm., 2013, 457, 150-157.

16 F. S. Palumbo, G. Pitarresi, C. Fiorica, P. Matricardi, A. Albanese and G. Giammona, Soft Matter, 2012, 8, 4918.

17 R. Fields, Methods Enzymol., 1972, 25, 464-468.

$18 \mathrm{H}$. Su, J. Han, Q. Dong, D. Zhang and Q. Guo, Nanotechnology, 2008, 19, 025601.

19 S. T. Ho and D. W. Hutmacher, Biomaterials, 2006, 27, 13621376.

20 T. Bitter and H. M. Muir, Anal. Biochem., 1962, 4, 330-334.

21 F. Braet, R. de Zanger and E. Wisse, J. Microsc., 1997, 186, 8487.

22 F. S. Palumbo, C. Fiorica, G. Pitarresi, M. Giorgi, F. Abramo, A. Gulino, M. Di Stefano and G. Giammona, J. Mater. Chem. $B, 2014,2,3243$.

23 Q. Lu, S. Zhang, K. Hu, Q. Feng, C. Cao and F. Cui, Biomaterials, 2007, 28, 2306-2313.

24 P. Zhou, X. Xie, D. P. Knight, X.-H. Zong, F. Deng and W.-H. Yao, Biochemistry, 2004, 43, 11302-11311.

25 K. Makaya, S. Terada, K. Ohgo and T. Asakura, J. Biosci. Bioeng., 2009, 108, 68-75.

26 R. Nazarov, H.-J. Jin and D. L. Kaplan, Biomacromolecules, 2004, 5, 718-726.

27 Q. Lu, X. Zhang, X. Hu and D. L. Kaplan, Macromol. Biosci., 2010, 10, 289-298.

28 M. Naeimi, M. Fathi, M. Rafienia and S. Bonakdar, J. Appl. Polym. Sci., 2014, 131, 41048-41056.

29 N. Bhardwaj, Q. T. Nguyen, A. C. Chen, D. L. Kaplan, R. L. Sah and S. C. Kundu, Biomaterials, 2011, 32, 5773-5781.

30 X. Z. Shu, Y. Liu, F. Palumbo and G. D. Prestwich, Biomaterials, 2003, 24, 3825-3834. 